\title{
Smoothing of Wind Farm Output by Prediction and Supervisory-Control-Unit-Based FESS
}

\author{
Farzana Islam, Member, IEEE, Ahmed Al-Durra, Member, IEEE, and S. M. Muyeen, Senior Member, IEEE
}

\begin{abstract}
This paper presents a supervisory control unit (SCU) combined with short-term ahead wind speed prediction for proper and effective management of the stored energy in a small capacity flywheel energy storage system (FESS) which is used to mitigate the output power fluctuations of an aggregated wind farm. Wind speed prediction is critical for a wind energy conversion system since it may greatly influence the issues related to effective energy management, dynamic control of wind turbine, and improvement of the overall efficiency of the power generation system. In this study, a wind speed prediction model is developed by artificial neural network (ANN) which has advantages over the conventional prediction schemes including data error tolerance and ease in adaptability. The proposed SCU-based control would help to reduce the size of the energy storage system for minimizing wind power fluctuation taking the advantage of prediction scheme. The model for prediction using ANN is developed in MATLAB/Simulink and interfaced with PSCAD/EMTDC. Effectiveness of the proposed control system is illustrated using real wind speed data in various operating conditions.
\end{abstract}

Index Terms-Flywheel energy storage system (FESS), induction generator (IG), power smoothing, supervisory control unit (SCU), wind speed prediction.

\section{INTRODUCTION}

B EING socially beneficial, economically competitive, and environment friendly, wind energy is now considered to be the world's fastest growing renewable energy. However, the stochastic nature of wind imposes a considerable challenge in the optimal management and operation of wind power generating systems. A larger number of wind turbine generators are going to be integrated into the power systems in the near future to meet the increasing demand of the loads. The intermittent nature of the wind speed is a serious issue for the power grid companies or transmission system operators (TSOs). As a result, it is essential to emphasize research on the power smoothing of the wind farm. Integrating an energy storage system (ESS) into the wind farm can mitigate the wind power fluctuation and hence improves the overall power quality of the wind farm output power. In general, the energy capacitor systems (ECS), superconducting magnetic energy storage systems (SMES), battery

Manuscript received August 08, 2012; revised December 05, 2012; accepted February 17, 2013. Date of publication April 25, 2013

The authors are with the Electrical Engineering Department, Petroleum Institute, Abu Dhabi, United Arab Emirates (e-mail: farzanaislam111@gmail.com). energy storage system (BESS), or flywheel energy storage systems (FESS), etc. [1]-[6] are used for the purpose of power smoothing. Since a fast response is necessary to compensate the output power fluctuations in the wind farm, battery storage systems might not be a good option because the charging and discharging of the battery is not so fast due to its chemical process [4]. In addition, it requires the same capacity power converters as the battery ratings. Frequent replacement of the battery cell is necessary as battery lifetime is not so long. ECS and SMES can also be used for power smoothing of wind farm due to their response speed and efficiency but their practical installation for large MW application is limited because of their higher maintenance cost.

Recently, FESS using rotational machines have become more popular because of their reliability, long life, large energy storage capacity, and less overall cost. FESS using the doubly-fed induction generator (DFIG) which is an adjustable speed generator, can improve the power system stability effectively. DFIG not only supplies the real power but also compensates reactive power demand rapidly and independently. Moreover, the small capacity power converter (typically $25 \%-30 \%$ of the total power) is required for DFIG operation and thus makes the overall system cost-effective [4]. Hence, the reactive power compensation of the system can be achieved at lower cost. It is also necessary to determine the capacity of the FESS required for smoothing the output power fluctuations effectively. It is expected that large capacity FESS $(H=50 \mathrm{~s})$ will give better performance in case of the output power smoothing [4]. But it will increase the system cost significantly. Therefore, a small capacity energy storage system requires having an efficient and proper energy management control strategy to keep the output power fluctuation of the wind farm within an acceptable range. Reducing the size of the FESS system is one of the objectives of this study.

Accurate prediction of wind speed is beneficial for power grid management, matching demand and supply, and in stabilization of the power system [7]. Precise prediction of wind speed accommodates the wind generating schedules in a wind farm efficiently. Numerous studies have been conducted on the accuracy of the predicted output both on long-term and short-term scenarios. The short-term wind speed prediction is extremely important for managing of energy reserve, pitch control mechanism, and changing gear for optimal wind turbine performance [8]. Several methods have been already proposed for the forecasting of the wind speed using different techniques such as persistence method, physical approach, statistical approach [8]-[25], etc. The statistical approach is based on training with measurement data and the difference between 
predicted and actual wind speeds in the immediate past to tune the model [10], [13]. Time series [14] and artificial neural network (ANN)-based [15]-[28] methods are classified as the statistical approach.

ANN simulates the human brain in processing information through a series of interconnected neurons and has an excellent ability of mapping complex and highly nonlinear input and output patterns without the actual model structure [15]. In ANN, the neurons are organized in layers, usually, an input, an output, and one or more hidden layers. Each of the neuron's connections has an adjustable weight. The feedforward network [16], multilayer perception network (MLP) [17]-[19], or recurrent neural network systems [20]-[22] are used extensively for wind speed prediction. Elman recurrent neural network (ERNN) is a kind of recurrent network (RNN) with great adaptability for time-varying patterns [23], [24]. Generally, compared with the static feedforward neural networks, the training for the ERNN is faster than the static feedforward neural networks. Radial basis function (RBF), owing to its superior performance of linear/ nonlinear algorithm with respect to fast convergence and accurate prediction, is also very suitable for wind speed prediction. RBF exhibits high approximation precision, and nonexistence of local minimum problems [25]-[28]. In the field of approximation capability, analysis ability, and learning speed, RBF neural network has superior performance compared to other artificial networks. Hence, this study is motivated to design a wind speed prediction model using ERNN and radial basis function neural network (RBFNN) followed by a comparative study between the two methods to develop a better control strategy in mitigating the output power fluctuation of wind farm.

Output power fluctuation of the wind farm can be effectively mitigated by a cooperative control of the wind farm and the FESS. In addition, use of an accurate prediction method like ANNs to predict the wind speed can be also applied to improve the overall power quality of the wind farm, especially for the power systems with small capacity energy storage systems. Because of the heavy inertia, a large capacity energy storage system mitigates random variation in the wind farm output power effectively. If the required power can be predicted and utilized properly to revise the final output power reference, then better performance can be achieved by reducing the size of the energy storage system and effective smoothing of the output power fluctuations can be achieved at lower cost. A new control strategy is necessary to provide the opportunities to improve the existing control strategies for enhancing the overall performances of the wind farm. Hence, in this study, a supervisory control unit (SCU) considering the advantage of short-term-ahead wind speed prediction scheme and small capacity energy storage system is developed to make the wind farm more reliable and efficient.

\section{MODEL SYSTEM}

An aggregated type wind farm [29] is considered in this study where multiple small size wind generators are represented with a large wind generator, mainly to speed up the simulation. The wind farm is composed of fixed speed wind turbine generator systems where an induction generator (IG) is used as the wind generator. The model system used for the simulation is shown in

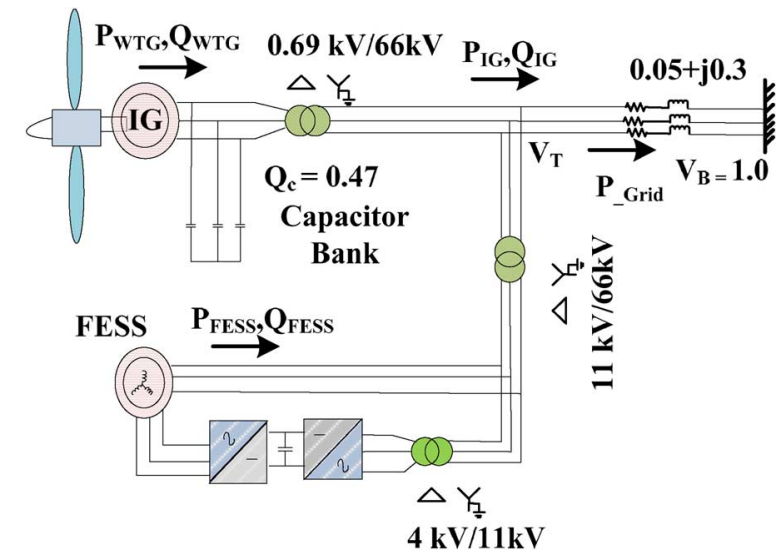

Fig. 1. Model system.

TABLE I

PARAMETERS FOR IG AND FESS [4]

\begin{tabular}{|l|l|l|}
\hline & IG & FESS \\
\hline Stator resistance (pu) & 0.01 & 0.02 \\
\hline Stator leakage reactance (p.u.) & 0.1 & 0.05 \\
\hline Magnetizing reactance (p.u.) & 3.5 & 4.01 \\
\hline Rotor resistance (p.u.) & 0.01 & 0.02 \\
\hline Rotor leakage reactance (p.u.) & 0.12 & 0.05 \\
\hline Inertia Constant, H (sec) & 1.5 & 15 \\
\hline
\end{tabular}

Fig. 1. The IG (10 MVA) is connected to an infinite bus through the transformer and a transmission line. A DFIG with the secondary excitation system is considered as FESS (4 MVA). The parameters used for IG and FESS are given in Table I.

\section{Modeling OF Wind Turbine AND Flywheel Energy STORAGE SYSTEM}

\section{A. Fixed Speed Wind Turbine Modeling}

The aerodynamic power captured by wind is given by

$$
P_{m}=\frac{1}{2} \rho C_{p}(\lambda) \pi R^{2} V_{w}^{3}
$$

where $P_{m}$ is the wind turbine output [W], $R$ is the radius of the blade [m], $\omega_{m}$ is the angular velocity of the wind turbine $[\mathrm{rad} / \mathrm{s}]$, $V_{w}$ is the wind speed $[\mathrm{m} / \mathrm{s}]$, and $\rho$ is the air density $\left[\mathrm{kg} / \mathrm{m}^{3}\right]$. The tip speed ratio $(\lambda)$ and power coefficient $\left(C_{p}\right)$ can be expressed as follows [30]-[34]:

$$
\begin{aligned}
\lambda & =\frac{\omega_{m} R}{V_{w}} \\
C_{p}(\lambda, \beta) & =\frac{1}{2}\left(\Gamma-0.02 \beta^{2}-5.6\right) e^{-0.17 \Gamma} \\
\Gamma & =\frac{R(3600)}{\lambda(1609)} .
\end{aligned}
$$

The torque coefficient $\left(C_{t}\right)$ and the turbine torque $T_{m}$

$$
\begin{aligned}
C_{t}(\lambda) & =\frac{C_{p}(\lambda)}{\lambda} \\
T_{m} & =\frac{1}{2} \rho C_{t}(\lambda) \pi R^{3} V_{w}^{2} .
\end{aligned}
$$




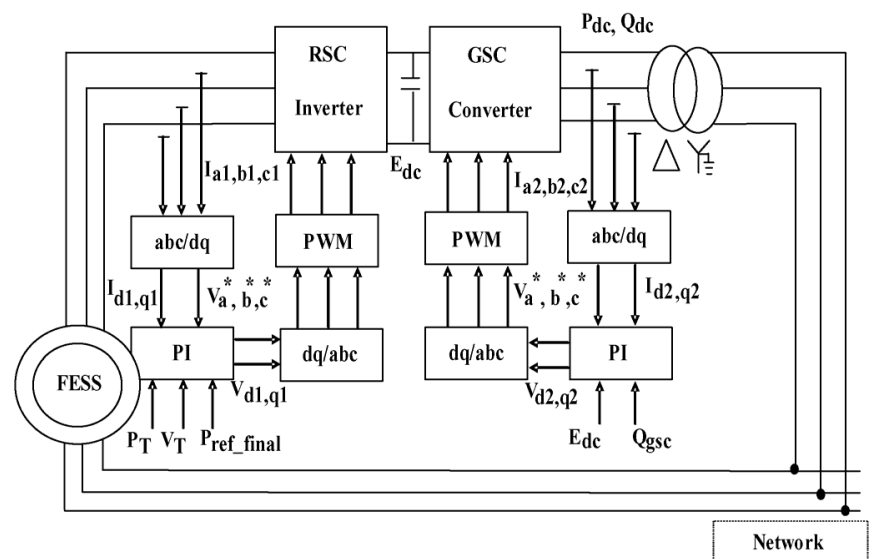

Fig. 2. Secondary excitation system of FESS.

\section{B. Configuration of FESS}

In FESS, the stator winding is connected directly to the grid and a bidirectional power converter is feeding the rotor winding. The power converter consists of two back-to-back IGBT bridges linked by a dc bus. The rotor side converter (RSC) is connected to the rotor winding. The inverter controls the active and reactive power output of the generator and the converter controls the dc link voltage and the reactive power that is flowing into the secondary excitation system. The dc link voltage is stabilized by the dc link capacitor and converted further by the grid side converter (GSC) into ac which is supplied to the grid. The secondary excitation system for FESS is shown is Fig. 2. The maximum and minimum energy that can be stored in FESS is limited to $\pm 30 \%$ of the rated energy. As shown in Fig. 2, the d-axis current $\left(I_{d 1}\right)$ in the RSC controls the active power of FESS while the q-axis current $\left(I_{q 1}\right)$ controls the reactive power of FESS. After $d q 0$ to $a b c$ transformation, $V_{d 1}^{*}$ and $V_{q 1}^{*}$ are sent to the PWM signal generator and three phase voltage, $V_{a b c^{*}}$ desired at the RSC are achieved. A sinusoidal PWM operation is carried out and switching signals are generated by applying triangular carrier wave comparison.

In GSC, d-axis current $\left(I_{d 2}\right)$ regulates the de link voltage at 1 p.u. while q-axis current $\left(I_{q 2}\right)$ controls the reactive power. After $d q 0$ to $a b c$ transformation, $V_{d 2}^{*}$ and $V_{q 2}^{*}$ are sent to the PWM signal generator and $V_{a b c^{*}}$ are the three phase voltage desired at the GSC. The carrier frequency for IGBT is chosen $2 \mathrm{kHz}$. Conventional PI controllers are used for converter and inverter control.

\section{Proposed Control Scheme for SMoothing OF WIND FARM}

A wind farm consisting of a large capacity FESS can smooth the output power fluctuation effectively because of its heavy inertia. But this increases the overall system cost. In order to achieve better smoothing effect using a small capacity FESS, it is essential to utilize the available energy in an optimum way. If the wind speed can be predicted in advance, based on this prediction, a proper control scheme can be designed to charge/ discharge of FESS more effectively.

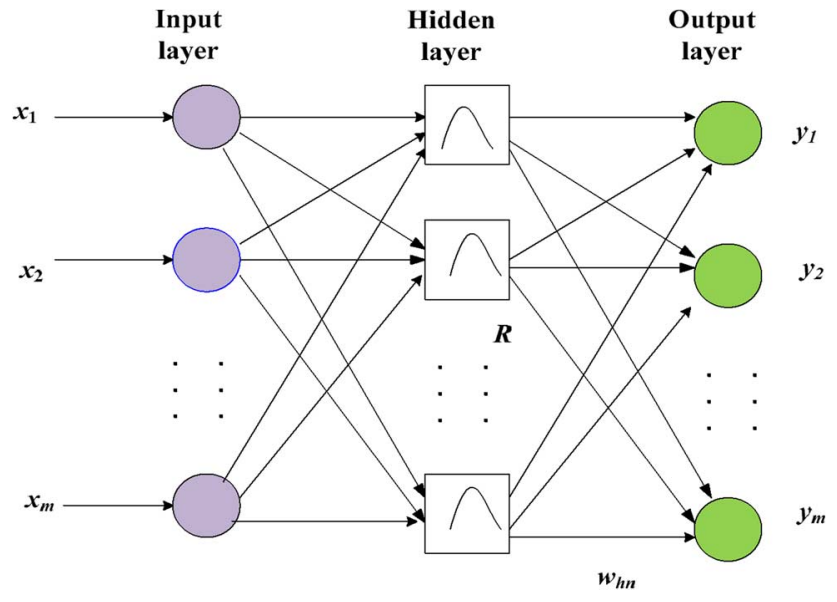

Fig. 3. Structure of RBFNN.

\section{A. Wind Speed Prediction by Using Artificial Neural Network}

In this study, two different kinds of neural networks, RBF and ERNN, have been modeled and analyzed for the purpose of wind speed prediction.

1) Radial Basis Function Neural Network (RBFNN): RBFNN is a feedforward network. The RBF network offers superior performance of linear/nonlinear algorithm with respect to fast convergence and accurate prediction and characterized by a combination of unsupervised (in the hidden layer) and supervised (in the output layer) training. The structure of RBF is shown in Fig. 3.

For the RBFNN learning algorithm, three parameters are necessary to be considered. They are the center of the base function, the mean square deviation, and the weights from the hidden layer to the output layer. The common RBF is a Gauss function in the RBFNN. From the structure of the RBFNN, the output of the network is given by [35]

$$
y_{i}=\sum_{i=1}^{h} w_{i j} \exp \left(-\frac{1}{2 \sigma_{j}^{2}}\left\|x_{p}-c_{i}\right\|^{2}\right), \quad j=1,2, \ldots, n
$$

where $x_{p}=\left(x_{1}^{p}, x_{2}^{p}, \ldots, x_{m}^{p}\right)^{T}$ is the $p$ th input sample, $p=$ $1,2, \ldots, P$, with $P$ is the total number of samples, $c_{i}$ is the center of hidden nodes, $w_{i j}$ is the connection weights from the hidden layer to the output layer, $i=1,2, \ldots, h$ is the number of hidden layer nodes, and $y_{j}$ is the real output of the $j$ th output node corresponding to the input sample.

2) Elman Recurrent Neural Network (ERNN): The ERNN is a partial recurrent network model which was first proposed by Elman [36]. The Elman network is two-layer back propagation network, with an addition of a feedback connection from the output of the hidden layer to its input. This feedback path allows the Elman network to learn to recognize and generate temporal patterns as well as spatial patterns [37].

The nonlinear state equation of the Elman network shown in Fig. 4 is built as follows [23]:

$$
\begin{aligned}
y(k) & =g\left(w_{2} x(k)\right) \\
x(k) & =f\left(w_{c} x_{c}(k)+w_{1} u(k-1)\right) \\
x_{c}(k) & =x(k-1)
\end{aligned}
$$




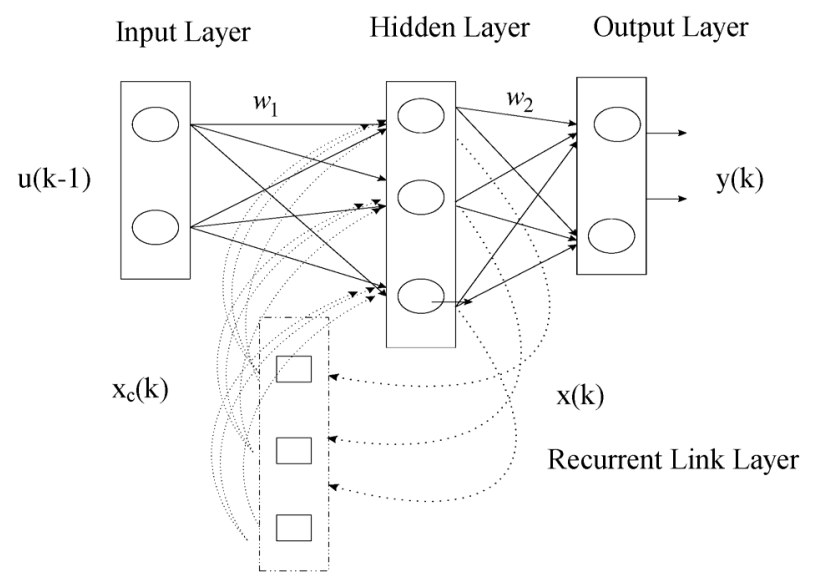

Fig. 4. ERNN model [23].

where $w_{c}, w_{1}, w_{2}$ are the weight values between recurrent link layer and input layer, weight values between input layer and hidden layer, and weight values between hidden layer and recurrent link layer, $u(k-1), x(k), y(k)$, and $x_{c}(k)$ are the scalar input, the values in hidden layer, the scalar output, and feedback values in the recurrent layer, respectively. $g($.$) and f($.$) are the$ transfer functions. Elman network has "tansig" neurons in its hidden layer and "purelin" in the output layer.

3) Prediction Results: For evaluating the wind speed prediction method, RBFNN and ERNN are implemented in MATLAB/Simulink. In this study, time series methodology is adopted where the past values of the process are used for estimating the future values after the model identification. Real wind speed data from Hokkaido Island, Japan has been used for simulation. The available wind speed data is partitioned into training set and test set. Here, 100 samples (each sample at every 3-s interval) are considered for the training purpose and the network model performance is evaluated by another 60 samples of data for both RBFNN and ERNN. In order to avoid the convergence problems during the training process, the network input data and corresponding target vector for the prediction model are normalized such that they fall within the range $[-1,1]$. In this study, the data is normalized by the following:

Normalized value $=$ Actual value/Maximum value.

The error function of $\mathrm{NN}$ is chosen to be mean squared error (MSE) of each pattern on the training set and given by

$$
\operatorname{MSE}=\frac{1}{N} \sum_{n=1}^{N}\left(x_{n}^{\text {actual }}-x_{n}^{\text {predicted }}\right)^{2} .
$$

A comparative study is carried out between RBFNN and ERNN methods using two different wind patterns (WPs) and three different network architectures (NAs). The network parameters used in different network architectures are given in Table II. The parameters relevant in the training algorithm, error goal, and number of epochs are chosen appropriately. RBFNN model is trained using "trainlm" (Lavenberg-Marquardt algorithm) and ERNN is trained with "traingdx" (gradient descent algorithm with adaptive momentum training algorithm) in
TABLE II

Wind Speed Prediction by Different Network ARchitectures

\begin{tabular}{|l|l|l|l|l|l|l|}
\hline $\begin{array}{l}\text { Network } \\
\text { parameters }\end{array}$ & \multicolumn{2}{|c|}{$\begin{array}{c}\text { Network } \\
\text { Architecture 1 } \\
\text { (NA1) }\end{array}$} & \multicolumn{2}{c|}{$\begin{array}{c}\text { Network } \\
\text { Architecture 2 } \\
\text { (NA2) }\end{array}$} & \multicolumn{2}{|c|}{$\begin{array}{c}\text { Network } \\
\text { Architecture 3 } \\
\text { (NA3) }\end{array}$} \\
\hline & RBFNN & ERNN & RBFNN & ERNN & RBFNN & ERNN \\
\hline Input & 3 & 2 & 5 & 4 & 4 & 3 \\
\hline Output & 1 & 1 & 1 & 1 & 1 & 1 \\
\hline $\begin{array}{l}\text { Neuron in } \\
\text { hidden } \\
\text { layer }\end{array}$ & 27 & 27 & 37 & 37 & 35 & 35 \\
\hline $\begin{array}{l}\text { MSE (\%) } \\
\text { WP1 }\end{array}$ & 40.8 & 51.5 & 40.29 & 24.3 & 11.57 & 15.43 \\
\hline $\begin{array}{l}\text { MSE (\%) } \\
\text { WP2 }\end{array}$ & 11.35 & 31.2 & 11.01 & 22.35 & 9.09 & 11.36 \\
\hline
\end{tabular}

the neural network training toolbox of MATLAB/Simulink. Here, for ERNN, the learning rate is varied from 0.1 to 0.35 . For RBFNN, the spread constant is varied between 0.1 and 0.7 , and the value giving the best predictive performance is chosen. Each network is simulated and their performance is observed by varying the number of inputs, number of hidden neurons, etc., as shown in Fig. 5. The error performances for all network architectures are also calculated using both wind patterns based on the MSE and summarized in Table II. It is found that RBFNN gives less error as compared to ERNN in all the architectures and among the three architectures; NA3 has the lowest MSE for both WP1 and WP2. Therefore, in this study, RBFNN is chosen to model the 3-min-ahead wind speed prediction.

\section{B. Supervisory Control Unit (SCU)}

An SCU shown in Fig. 6 helps the proposed control scheme to adjust the output power reference of FESS considering the rotor speed $\left(\omega_{r}\right)$ of FESS, actual energy stored in FESS $\left(E_{a}\right)$, actual wind speed $\left(V_{a}\right)$, and predicted wind speed $\left(V_{p}\right)$. Here, "Flag" is another input variable which indicates whether the output of WF is in charging mode or discharge mode. $P_{\text {diff }}$ indicates the power difference between the actual and predicted wind power. The output power reference $1\left(P_{\text {ref }_{-} 1}\right)$ is generated using a low pass filter function where the input of the filter function is output power from wind generator $P_{\mathrm{IG}}$. In order to apply the short-term-ahead $(3 \mathrm{~min})$ wind speed prediction model by ANN into the proposed control scheme, the time constant, T1 for the filter function is chosen to be $180 \mathrm{~s}$. When the output of the IG is higher than the final output power reference, FESS is in charging mode and absorbs the fluctuating part of wind farm output power. But when the generated output is lower than the final output power reference, FESS is in discharge mode and supplies active power to the grid. However, when the stored energy reaches close to its maximum limit, output power reference for the system is modified in such a way that the stored energy is forced to be in discharge mode so that it does not exceed its maximum limit anymore. Similarly, when the FESS almost reaches its minimum limit, then the reference is corrected in such a way that it is forced back to charging mode again.

An efficient energy management in the FESS is configured considering different operating cases. Higher switching frequencies of SCU are eliminated by filtering it through a low 


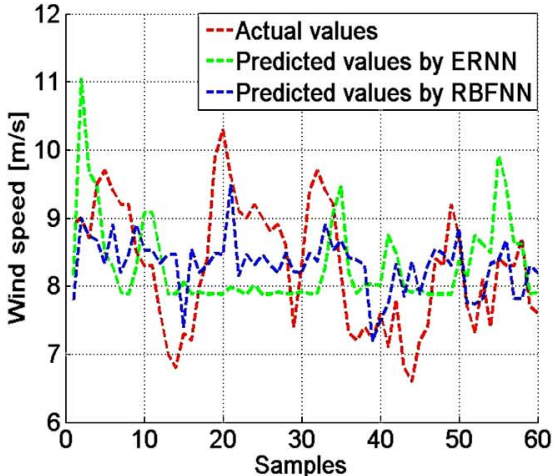

(a)

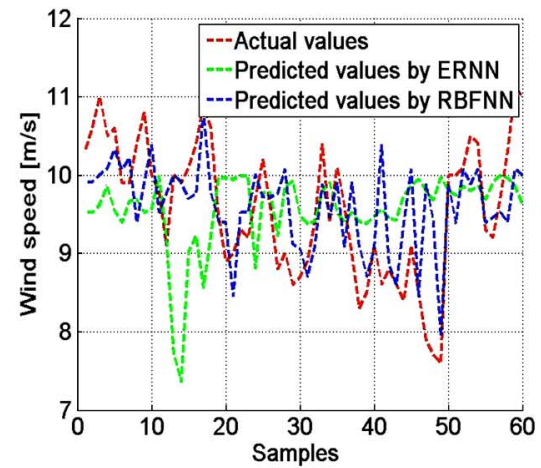

(d)

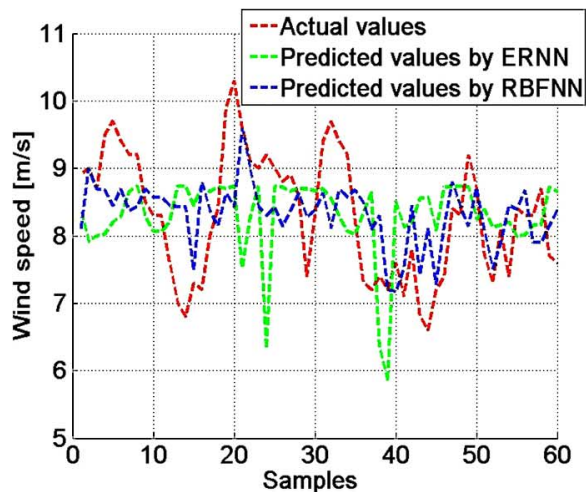

(b)

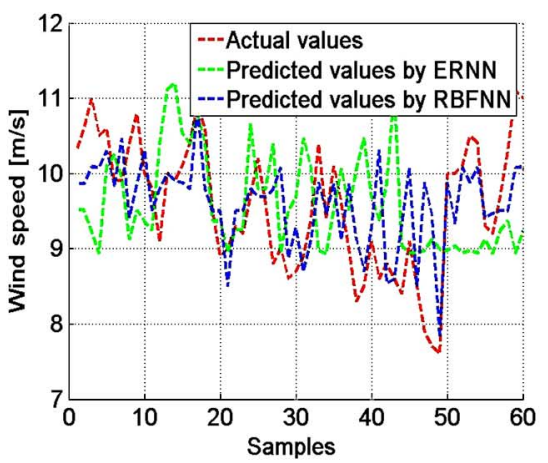

(e)

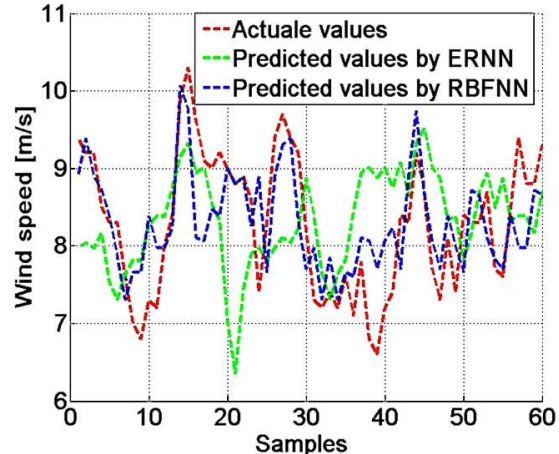

(c)

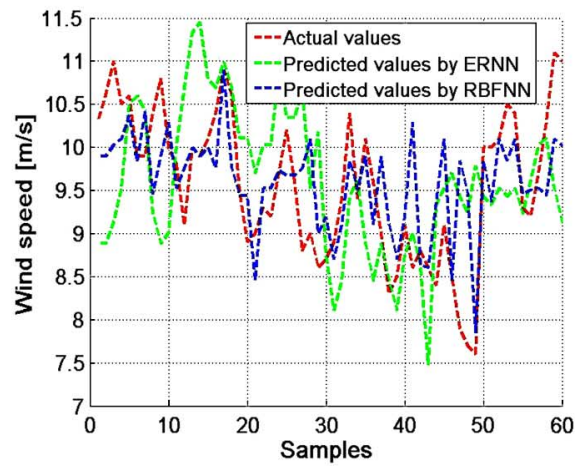

(f)

Fig. 5. Wind speed prediction using (a) NA1 and WP1, (b) NA2 and WP1, (c) NA3 and WP1, (d) NA1 and WP2, (e) NA2 and WP2, and (f) NA3 and WP2.

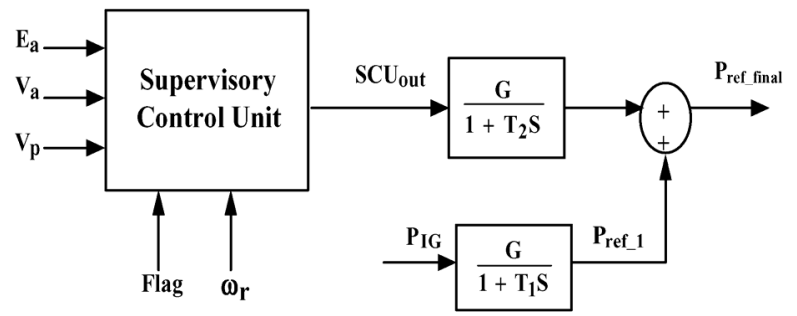

Fig. 6. Reference signal generation using SCU for the proposed control scheme.

pass filter with a time constant $\left(T_{2}\right)$ of $5 \mathrm{~s}$. Different operating conditions for SCU are shown in Fig. 7 and explained as follows.

Case $1\left(\omega_{r} \geq 1.28\right)$ : This case indicates that the stored energy in FESS is going to reach its maximum level. As a result, the final output power reference has to be modified in such a way that no more energy will be stored in the FESS.

In this case, the final output power reference will be shifted up above the output of wind farm $P_{\mathrm{IG}}$ so that the FESS is in discharging mode and thus FESS will be prevented from charging any more. The output power reference is set by $\mathrm{SCU}_{\text {out }}=$ $\left(E_{a}-0.75\right)^{*} g 1$.

Case $2\left(\omega_{r} \geq 1.25 \&\left(V_{p}>V_{a}\right) \&\right.$ Flag $\left.=1\right)$ : This case indicates that FESS is already charging and if it continues to charge then it will reach its maximum level. Therefore, only discharging of FESS is allowed for this situation. In order to discharge, the final output power reference is set by $\mathrm{SCU}_{\text {out }}=$ $\left(E_{a}-0.75\right)^{*}$ gain. The value of gain is chosen depending on the value of the $P_{\text {diff }}$, as shown in the Fig. 7. If $\left(P_{\text {diff }} \geq 0.5\right)$, then the value of gain will be $g 2$, and if $\left(P_{\text {diff }} \leq 0.15\right)$, then the value of gain will be $g 3$.

Case $3\left(\omega_{r} \geq 1.2 \&\left(V_{p}>V_{a}\right) \&\right.$ Flag $\left.=0\right)$ : This case indicates that FESS has not reached its maximum energy level yet. So charging/discharging of FESS is allowed without any restriction. The output power reference is set by $\mathrm{SCU}_{\text {out }}=$ $\left(E_{a}-0.75\right)$.

Case $4\left(0.85 \leq \omega_{r}<1.2\right)$ : In this state, the FESS is in normal operating range. So there is no restriction. The power fluctuation is mitigated by the stored energy in FESS.

Case $5\left(\omega_{r}<0.85 \&\left(V_{p}<V_{a}\right) \&\right.$ Flag $\left.=1\right)$ : This case also indicates that the minimum level of FESS has not been accomplished yet and there is no restriction for charging or discharging. So the output power reference will be the same as discussed in Case 3.

Case $6\left(\omega_{r}<0.85 \&\left(V_{p}>V_{a}\right) \&\right.$ Flag $\left.=0\right)$ : This case indicates that the FESS is already in discharging mode. So, if it continues to discharge then it will reach to its minimum limit. That is why the control for output power reference is set to charge forcefully. This is done by setting output power reference, $\mathrm{SCU}_{\text {out }}=\left(E_{a}-0.75\right)^{*}$ gain. The value of gain will be chosen depending on the value of $P_{\text {diff }}$ as explained in Case 2.

Case $7\left(\omega_{r} \leq 0.75\right)$ : This case indicates that the FESS is operating almost at minimum stored energy level. In order to avoid any instability in the system, the output power reference is forcefully set to charge by setting the value for $\mathrm{SCU}_{\text {out }}=$ $\left(E_{a}-0.75\right)^{*} g 4$.

The value of gain varies depending on the difference of actual and predicted wind speed. Table III shows the values for 


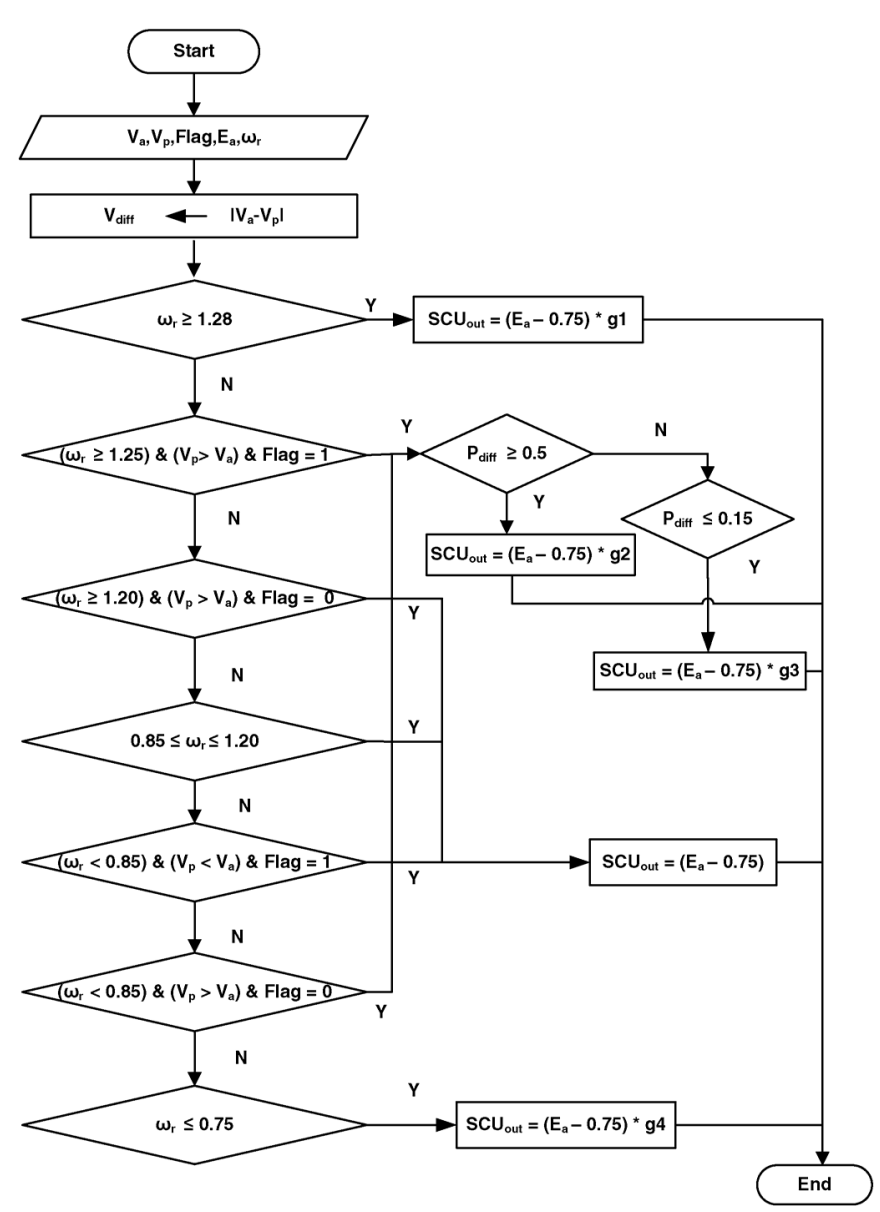

Fig. 7. Flowchart for control signal generation using SCU.

TABLE III

DIFFERENT VALUES OF GAIN

\begin{tabular}{|c|c|c|c|}
\hline g1 & g2 & g3 & g4 \\
\hline 2.2 & 2 & 0.7 & 0.15 \\
\hline
\end{tabular}

different gains which are used in Fig. 7. The effectiveness of the proposed system is verified by simulation results.

\section{Interfacing Between MATLAB/Simulink and PSCAD/EMTDC}

In this study, the model for wind speed prediction is developed using ANN in MATLAB/Simulink and it is used from PSCAD/EMTDC software where the model system including the control scheme is developed. The MATLAB interface feature is not supported by the default GNU FORTRAN complier used in PSCAD. Hence, Intel FORTRAN Compiler V 9.0 has to be used to interface PSCAD/EMTDC and MATLAB/Simulink. The key subroutine for using the MATLAB interfacing feature is "MLAB_INT." To speed up the overall simulation, an impulse generator is used with an enable/disable pulse.

\section{Simulation Results}

Fig. 8 shows the real wind speed data and 3-min-ahead predicted wind speed data which are used for simulation in PSCAD/EMTDC. From this figure, it is seen that the wind speed fluctuates between a high wind speed and low wind speed

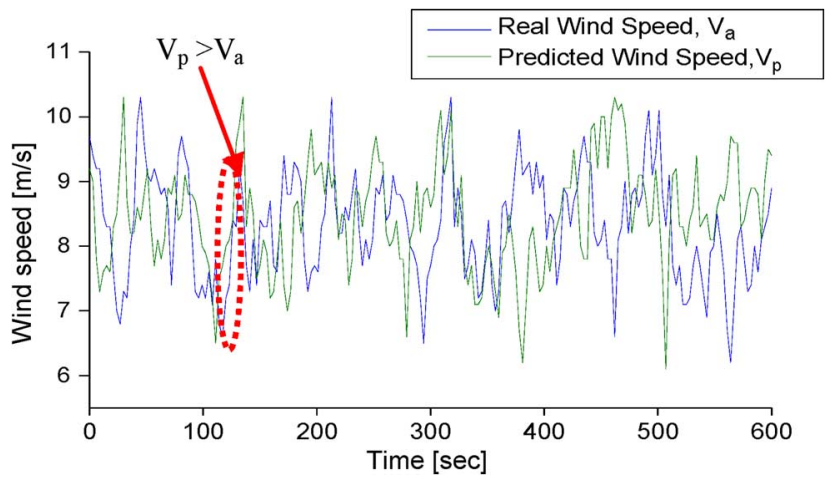

Fig. 8. Real and 3-min-ahead predicted wind speed data.

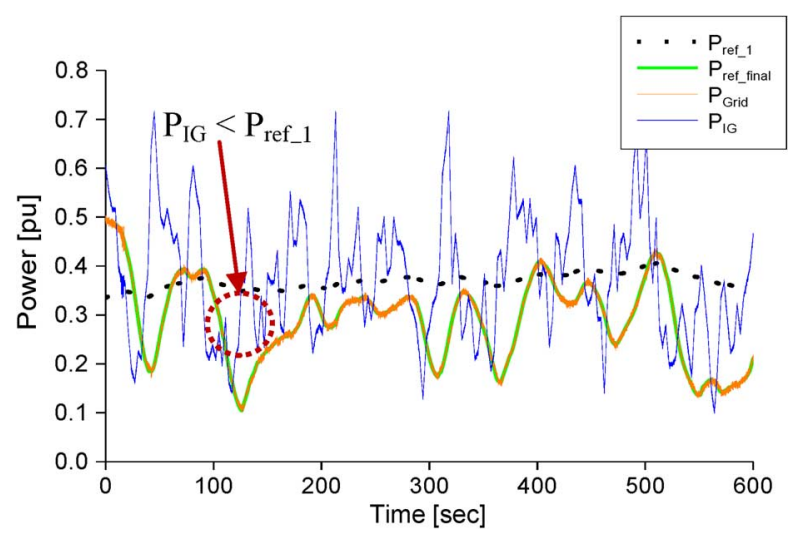

Fig. 9. Output power reference 1; final output power reference; grid output power; WF output power [p.u.].

in a short period. The time step and run time for the simulation are chosen $20000 \mu \mathrm{s}$ and $600 \mathrm{~s}$, respectively. It is also found that a small capacity energy storage system with $H=15 \mathrm{~s}$ is not sufficient enough to smoothen the power fluctuation as well as maintaining system stability if the final output power reference is set to $P_{\text {ref_1 } 1}$. The overall system will become unstable if the final output power reference is not modified according to the proposed SCU scheme. Fig. 9 shows the wind generator output power, the smoothed grid power, output power reference 1 , and the final reference power using the proposed control scheme. The wind generator output power varies according to the wind speed. In Fig. 9, it is seen that $P_{- \text {Grid }}$ and $P_{\text {ref } \_ \text {final }}$ are overlapped as FESS properly charges and discharges to follow the reference command signal shown in Fig. 2. The stored energy level and the rotor speed of FESS shown in Figs. 10 and 11, respectively, are controlled by the control of SCU. For example, in Fig. 9, at around $110 \mathrm{~s}$, the FESS is supposed to be in discharging mode as the output of $P_{\mathrm{IG}}$ is at lower level than the $P_{\text {ref_1 }}$. However, at the same instant, it is observed in Fig. 8 that $V_{p}$ is also higher as compared to $V_{a}$. In this situation, if FESS continues to discharge normally, then it will exceed its minimum limit and the system will collapse as DFIG is not allowed to be operated at a speed which is less than $30 \%$ from the rated speed. As a result, SCU sets the command in such a way that it starts charging forcefully up to a certain limit. Hence, the FESS is prevented from reaching its minimum limit as mentioned in Case 6. The output of the SCU for different 


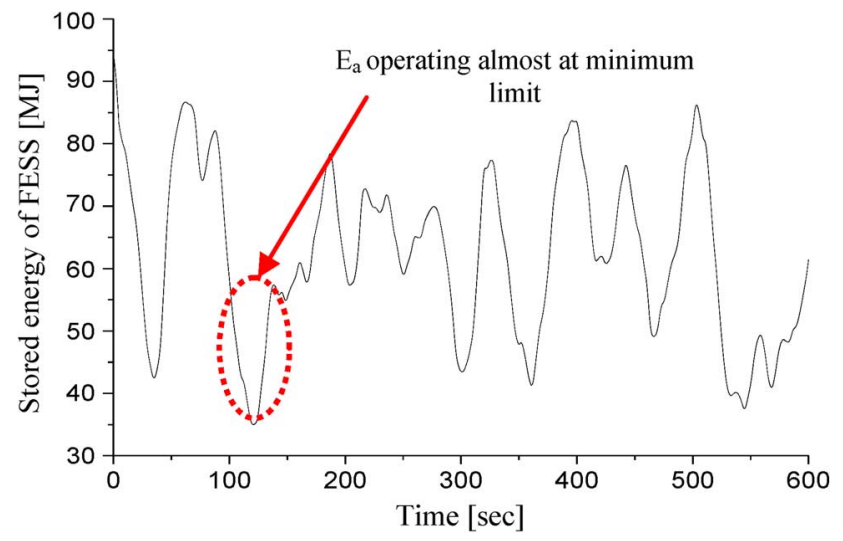

Fig. 10. Stored energy in FESS [MJ].

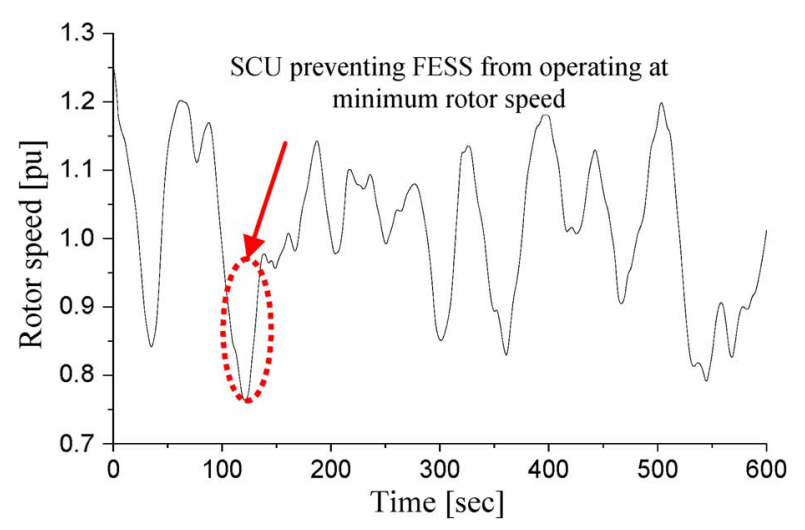

Fig. 11. Rotor speed of FESS [p.u.].

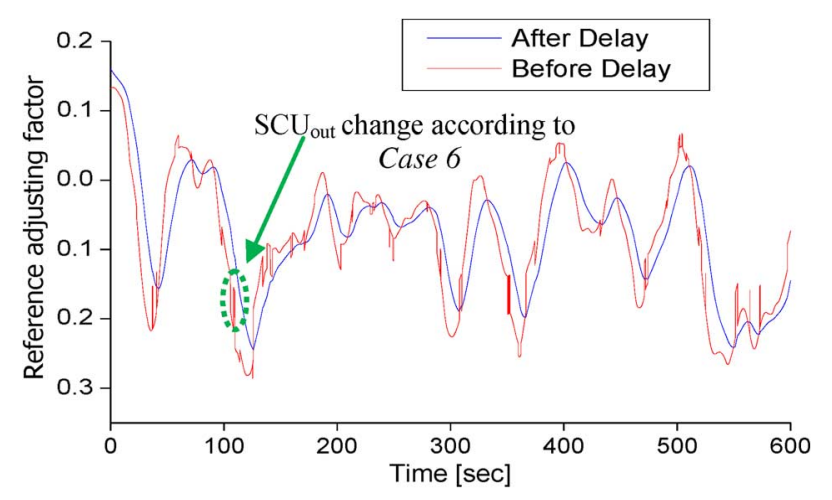

Fig. 12. Output of SCU.

operating condition has been demonstrated in Fig. 12. Higher switching frequencies of the signal $\mathrm{SCU}_{\text {out }}$ are omitted by passing it through a first-order delay function. Response for active power of FESS is shown in Fig. 13. The FESS supplies or absorbs the real power according to the output power reference and hence smooths the total power supplied to the grid. The dc link voltage $\left(E_{\mathrm{dc}}\right)$ and the terminal voltage $\left(V_{T}\right)$ of the system are maintained constant at 1.0 p.u., as shown in Figs. 14 and 15, respectively. The benefit of the proposed control scheme lies in the fact that the rotor speed of FESS is operating in between its normal operating range which is defined as $\left(0.7 \leq \omega_{r} \leq 1.3\right)$.

Due to the proper energy storage management with SCU, the overall system is still able to supply the output power to the grid

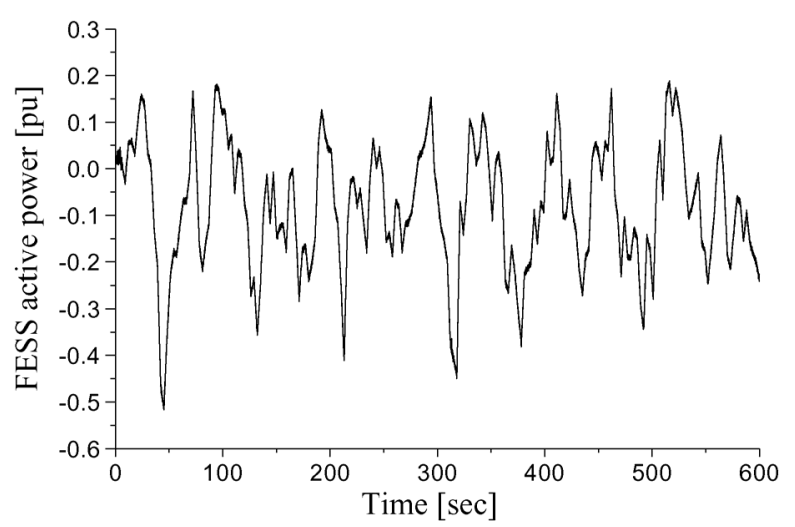

Fig. 13. Active power of FESS [p.u.].

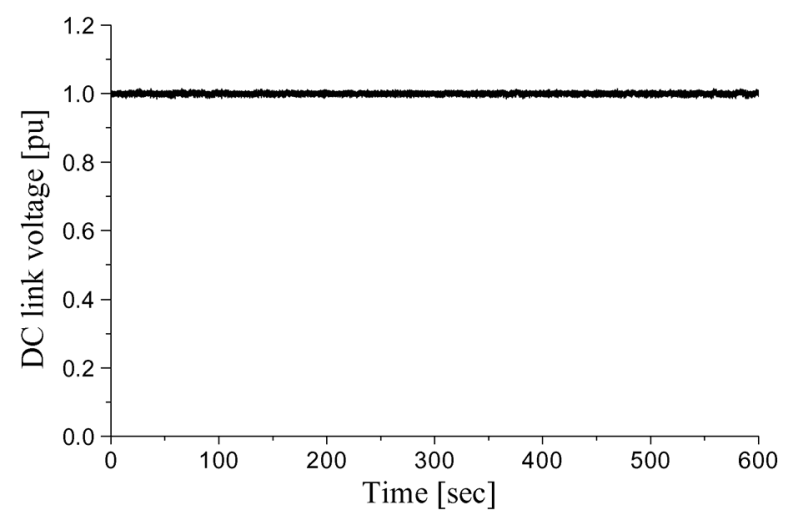

Fig. 14. DC link voltage [p.u.].

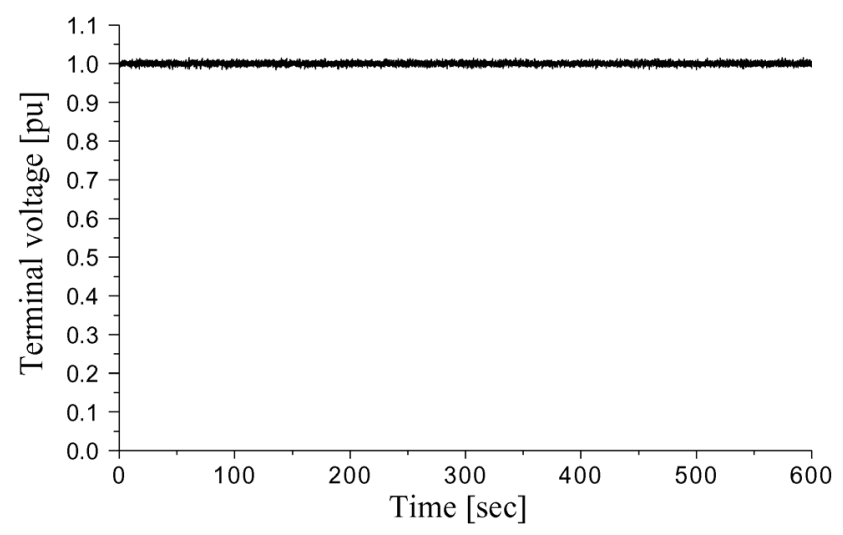

Fig. 15. Terminal voltage [p.u.].

even though the performance deteriorates when FESS operated at its maximum/minimum speeds.

\section{CONCLUSION}

A coordinated control strategy combined with short-termahead wind speed prediction and a small capacity SCU-based FESS has been proposed in this study to reduce the output power fluctuations of the grid connected wind farm. RBFNN and ERNN both are modeled for the purpose of wind speed prediction. RBFNN is found to perform better compared to the performance of ERNN and has been chosen to carry out simulation and evaluate the performance of the proposed control strategy. The model for wind speed developed in 
MATLAB/Simulink is interfaced with PSCAD/EMTDC. Simulation results verify that taking the benefits of prediction of the wind speed and modification of output power by an SCU makes the small capacity FESS more effective and smooths the output power fluctuations more efficiently.

\section{ACKNOWLEDGMENT}

F. Islam would like to thank Electric Machine Laboratory, Kitami Institute of Technology, Japan for providing the real wind speed data which has been used for simulation.

\section{REFERENCES}

[1] S. M. Muyeen, R. Takahashi, T. Murata, and J. Tamura, "Integration of an energy capacitor system with a variable-speed wind generator," IEEE Trans. Energy Convers., vol. 24, no. 3, pp. 740-749, Sep. 2009.

[2] F. Zhou, G. Joos, C. Abbey, L. Jiao, and B. T. Ooi, "Use of large capacity SMES to improve the power quality and stability of wind farms," in Proc. IEEE Power Eng. Soc. Gen. Meeting, Jun. 2004, vol. 2, pp. 2025-2030.

[3] S. M. Muyeen, M. H. Ali, R. Takahashi, T. Murata, and J. Tamura, "Wind generator output power smoothing and terminal voltage regulation by using STATCOM/ESS," in Proc. IEEE Power Tech. 2007 Conf., Lausanne, Switzerland, Jul. 2007, pp. 1232-1237, Paper 258.

[4] R. Takahashi and J. Tamura, "Frequency control of isolated power system with wind farm by using flywheel energy storage system," in Proc. 18th Int. Conf. Electrical Machines, Vilamoura, Portugal, Sep. 2008, pp. 1-6.

[5] R. Hebnor, J. Beno, and A. Walls, "Flywheel batteries come around again," IEEE Spectrum, vol. 39, no. 4, pp. 46-51, Apr. 2002.

[6] G. O. Cimuca, C. Saudemont, B. Robins, and M. M. Radulescue, "Control and performance evaluation of a flywheel energy storage system associated to a variable - Speed wind generator," IEEE Trans. Ind. Electron., vol. 53, no. 4, pp. 1074-1084, Aug. 2006.

[7] X. Wang, G. Siderators, N. Hatziargyrion, and L. H. Tsoakalas, "Wind speed forecasting for power system operational planning," in Proc. Int. Conf. Probabilistic Methods applied to Power System, Sep. 12-16, 2004, pp. 470-474.

[8] S. Dutta and T. J. Overbye, "Prediction of short term power output of wind farms based on least squares method," in Proc. IEEE Power and Energy Society General Meeting, Jul. 2010, pp. 1-6.

[9] Y. K. Wu and J. S. Hong, "A literature review of wind forecasting technology in the world," in Proc. IEEE Power Tech 2007, Lausanne, Switzerland, Jul. 2007, pp. 504-504.

[10] C. W. Potter and M. Negnevitsky, "Very short term wind forecasting for Tasmanian Power Generation," IEEE Trans. Power Syst., vol. 21, no. 2, pp. 965-972, May 2006.

[11] A. Costa, A. Crapo, and J. Navarro, "A review on the young history of the wind power short-term prediction," Renew. Sustain. Energy Rev., vol. 12, no. 6, pp. 1725-1744, Aug. 2008.

[12] B. Candy, S. J. English, and S. J. Keogh, "A comparison of the impact of QuikScat and WindSat wind vector products on met office analyses and forecasts," IEEE Trans. Geosci. Remote Sens., vol. 47, no. 6, pp. 1632-1640, Jun. 2009.

[13] M. Lange and U. Focken, "New developments in wind energy forecasting," in Proc. IEEE Power and Energy Society General Meeting 2008-Conversion and Delivery of Electrical Energy in the 21st Century, Pittsburg, PA, USA, Jul. 2008, pp. 1-8.

[14] J. L. Torres, A. Garcia, M. De Blas, and A. De Francisco, "Forecast of hourly average wind speed with ARMA models in Navarre (Spain)," Solar Energy, vol. 79, no. 1, pp. 65-77, Jul. 2005.

[15] S. N. Pandey, S. Tapawsi, and L. Srivastava, "Nodal congestion price estimation in spot power market using artificial neural network," IET Generat., Trans., Distrib., vol. 2, no. 2, pp. 280-290, Mar. 2008.

[16] M. Mohandes, S. Rehman, and T. Halawani, "A neural network approach for wind speed prediction," Renew. Energy, vol. 13, no. 3, pp. 345-354, Mar. 1998.

[17] J. J. Rubio, P. Angelov, and J. Pacheco, "Uniformly stable backpropagation algorithm to train a feedforward neural network," IEEE Trans. Neural Netw., vol. 22, no. 3, pp. 356-366, Mar. 2011.

[18] W. Zhang, W. Wu, and M. Yao, "Boundedness and convergence of bath backpropagation algorithm with penalty with feedforward neural networks," Neurocomputing, vol. 89, pp. 141-146, 2012.
[19] J. J. Rubio, "Modified optimal control with a backpropagation network for robotic arms," IET Control Theory Applicat., vol. 6, no. 14, pp. 2216-2225, 2012

[20] J. H. Perez-Cruz, J. J. Rubio, E. ruiz-Velazquez, and G. Solis-Perales, "Tracking control based on recurrent neural networks for nonlinear systems with multiple inputs and unknown deadzone," Abstract Appl. Anal., vol. 2012, pp. 1-18, 2012.

[21] X. Wang and Y. Huang, "Convergence study in a extended Kalman filter-based training of recurrent neural networks," IEEE Trans. Neural Netw., vol. 22, no. 4, pp. 588-600, Apr. 2011.

[22] C. K. Ahn, "Robust stability of recurrent neural networks with ISS learning algorithm," Nonlinear Dynamics, vol. 65, no. 4, pp. 413-419, 2011.

[23] J. Li, B. Zhang, C. Mao, G. Xie, Y. Li, and J. Lu, "Wind speed prediction based on the Elman recursion neural networks," in Proc. 2010 Int. Conf. Modeling, Identification and Control, Okayama, Japan, Jul. 2010 , pp. $728-732$.

[24] L. Xiang, C. Zengqiang, and Y. Zhuzhi, "Non linear stable adaptive control based upon Elman networks," Appl. Math.-J. Chinese Univ., vol. 15 , no. 3, pp. 332-340, Sep. 2000.

[25] X. Yong-Shan, W. Wei-Qing, and H. Xiao-ping, "Study on the time series wind speed forecasting of the wind farm based on neural networks," Energy Convers. Technol., vol. 25, no. 142, pp. 106-108, Mar. 2007.

[26] W. Junli, L. Xingjie, and Q. Jian, "Wind speed and power forecasting based on RBF neural network," in Proc. Int. Conf. Computer Application and System Modeling (ICCASM), Oct. 2010, vol. 5, pp. 298-301.

[27] P. Angelov, "Fuzzily connected multimodel systems evolving autonomously from data streams," IEEE Trans. Syst., Man, Cybern. B, Cybern., vol. 41, no. 4, pp. 898-910, Aug. 2011.

[28] A. Lemos, W. Caminhas, and F. Gomide, "Multivariable Gaussian evolving fuzzy modeling system," IEEE Trans. Fuzzy Syst., vol. 19, no. 1, pp. 91-104, Feb. 2011

[29] I. Zubia, X. Ostolaza, G. Tapia, A. Tapia, and J. R. Saenz, "Electrical fault simulation and dynamic response of a wind farm," in Proc. IASTED Int. Conf. Power and Energy Systems, 2001, pp. 595-600.

[30] C. Y. Tang, Y. Guo, and J. N. Jiang, "Nonlinear dual-mode control of variable-speed wind turbines with doubly fed induction generators," IEEE Trans. Control Syst. Technol., vol. 19, no. 4, pp. 744-756, Jul. 2011.

[31] J. J. Rubio, M. Figueroa, J. Pacheco, and M. Jimenes-Lizarraga, "Observer design based in the mathematical model of a wind turbine," Int J. Innovative Comput., Inf., Control, vol. 7, no. 12, pp. 6711-6725, 2011.

[32] R. Vepa, "Nonlinear, optimal control of a wind turbine generator," IEEE Trans. Energy Convers., vol. 26, no. 2, pp. 468-478, Jun. 2011.

[33] O. Wasynczuk, D. Man, and J. P. Sullivan, "Dynamic behavior of a class of wind turbine generators during random wind fluctuations," IEEE Trans. Power App. Syst., vol. PAS-100, no. 6, pp. 2854-2854, 1981.

[34] R. Takahashi, J. Tamura, M. Futami, M. Kimura, and K. Ide, "A new control method for wind energy conversion system using double-fed synchronous generators," (in Japanese) IEEJ Trans. Power Energy, vol. 126 , no. 2, pp. 225-235, 2006.

[35] X. Wu, F. Wen, B. Hong, X. Peng, and J. Huang, "Radial basis function neural network based short-term wind power forecasting with Grubbs Test," in Proc. IEEE 4th Int. Conf. Electric Utility Deregulation and Restructuring and Power Technologies (DRPT), Weihai, China, Jul. 2011, pp. 1879-1882.

[36] J. L. Elman, "Finding structure in time," Cognitive Science, vol. 14, no. 2, pp. 179-211, 1990.

[37] M. Hagan, H. Demuth, and M. Beale, Neural Network Design. : :PWS Publishing Company and Thomson Asia, 2002.

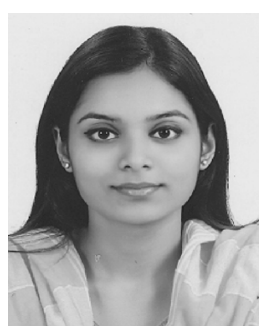

Farzana Islam (S'11-M'12) received the B.Sc. degree from American International University-Bangladesh (AIUB), in 2008, and the M.Sc. degree from The Petroleum Institute, Abu Dhabi, U.A.E., in 2012.

She was working as a Lecturer in the Electrical and Electronic Engineering Department, American International University-Bangladesh (AIUB) from 2008 to 2010 . Her research interests are energy storage system (ESS) and renewable energy. 


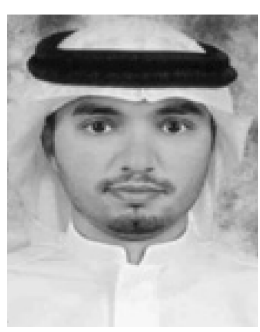

Ahmed Al-Durra (S'07-M'10) received the B.S., M.S., and Ph.D. degrees in electrical and computer engineering from the Ohio State University in 2005, 2007, and 2010, respectively. For his M.Sc. degree, he investigated the application of several nonlinear control techniques on automotive traction PEM fuel cell systems. He conducted his Ph.D. research at the Center for Automotive Research in the Ohio State University. His Ph.D. work was on the applications of modern estimation and control theories to automotive propulsion systems.

At the present, he is working as Assistant Professor in the Electrical Engineering Department, Petroleum Institute, Abu Dhabi. His research interests are application of estimation and control theory in power system stability and control, energy storage system (ESS), and renewable energy.

Dr. Ahmed is a member of ASME.

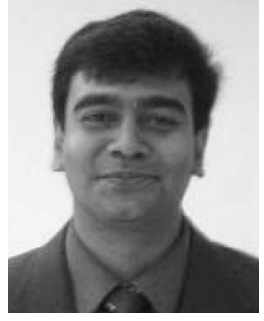

S. M. Muyeen (S'03-M'08-SM'12) received the B.Sc.Eng. degree from Rajshahi University of Engineering and Technology (RUET), Bangladesh, formerly known as Rajshahi Institute of Technology, in 2000, and the M.Sc.Eng. and Dr.Eng. degrees from Kitami Institute of Technology, Japan, in 2005 and 2008, respectively, all in electrical and electronic engineering. His Ph.D. research work focused on wind farm stabilization from the viewpoint of LVRT and frequency fluctuation.

After completing his Ph.D. program, he worked as a Postdoctoral Research Fellow under the versatile banner of Japan Society for the Promotion of Science (JSPS) from 2008 to 2010 at the Kitami Institute of Technology, Japan. At the present, he is working as Assistant Professor in the Electrical Engineering Department, Petroleum Institute, Abu Dhabi. His research interests are power system stability and control, electrical machine, FACTS, energy storage system (ESS), renewable energy, and HVDC system. He has published over 100 international papers. He has published five books as an author or editor.

Dr. Muyeen is the member of IEEJ. 